\title{
The Analysis of Liquid Metal Flow Characteristics in the Annular Passage of an Electromagnetic Pump
}

\author{
Chang Eob Kim $†$, Mun Ho Jeon*, Jeong Tae Kwon**, Hyo Jae Lim** and Suk Won Lee***
}

\begin{abstract}
An electromagnetic pump using a tubular induction motor (TLIM) has been proposed to pump liquid metal fluids. TLIM has been designed for liquid metal flow systems with a motor with a thrust force of 40 77[N]. The flow characteristics have been investigated by solving the Navier-Stokes equation, where the Lorentz force was included simply by considering it as a constant in the NavierStokes equation. A wood metal was chosen to simulate the liquid metal. The effect of Lorentz force on the flow rate was investigated. An experiment was conducted and its results were compared with those of the simulation. The simulation result showed an overestimation of about $17 \%$ compared with the experimental one.
\end{abstract}

Keywords: Electromagnetic pump, TLIM, Lorentz force, Navier-Stokes equation, Liquid metal

\section{Introduction}

The linear induction motor (LIM) has been widely used in various fields [1]-[3]. As an application of an LIM, an electromagnetic pump using a tubular linear induction motor (TLIM) has been proposed. The force of the LIM pump is produced by the interaction between the traveling magnetic fields and induced current of the molten metal, which is used to transfer the molten metal without any contact. Consequently, the flow characteristics of molten metal have to be solved by coupling the electromagnetic equation with fluid dynamics. In this paper, the flow characteristics of the molten metal are analyzed for various conditions and the simulation results are investigated. The wood metal, which is composed of $\mathrm{Bi}, \mathrm{Pb}, \mathrm{Sm}, \mathrm{Cd}$, is selected as a molten metal as it can be handled easily in the laboratory due to its low melting point. Fig. 1 is the schematic diagram for

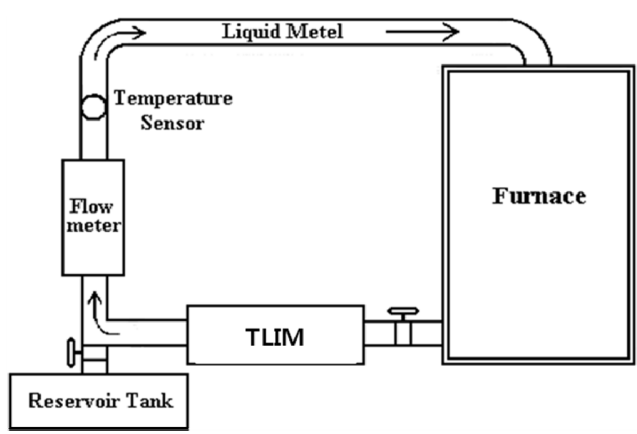

Fig. 1. The schematic diagram of the fluid system.

$\dagger$ Corresponding Author: Department of Electrical Engineering, Hoseo University, Korea. (cekim@hoseo.edu)

* Department of Mechanical Engineering, Hoseo University, Korea. (munho76@hanmail.net)

** Department of Mechanical Engineering, Hoseo University, Korea. (jtkwon@hoseo.edu,hjlim@hoseo.edu)

*** Department of System Control Engineering, Hoseo University, Korea. (swlee@hoseo.edu)

Received: March 12, 2009; Accepted: March 31, 2010 the fluid system. The fluid system is composed of a TLIM, a flow meter to measure the flow velocity, and a furnace to melt the metal. In the design of the electromagnetic pump, the targets of fluid speed and flow rate are $0.29[\mathrm{~m} / \mathrm{s}]$ and $15[\ell / \mathrm{min}]$, respectively. The corresponding TLIM was designed for the fluid system; the thrust force of $40 \sim 77[\mathrm{~N}]$ for AC 220[V], 60[Hz]. Then the flow characteristics of the system with TLIM were analyzed and compared with the target values.

\section{Design of TLIM for Electromagnetic Pump}

For the pumping flow rate $Q$, the pressure drop $\Delta P$ and the average flow velocity $V$, the necessary driving power of TLIM is calculated as follows [4], [5]:

$$
\begin{aligned}
& P_{d}=\Delta P \cdot Q=F_{x} \cdot V \\
& \Delta P=\rho \cdot g \cdot h \\
& h=\frac{V^{2}}{2 g}+0.5 \frac{V^{2}}{2 g}+1.2 \frac{V^{2}}{2 g}+f \frac{L}{D} \frac{V^{2}}{2 g}+\Delta h
\end{aligned}
$$

Where, $\rho$ is fluid density[kg.m $\left.{ }^{3}\right], g$ gravity $\left[9.8 \mathrm{~kg} / \mathrm{s}^{2}\right]$, $Q$ flow rate[ $\ell / \mathrm{min}], F_{x}$ thrust force[N], $V$ fluid speed $[\mathrm{m} / \mathrm{s}], h$ total head[m], $\Delta h$ elevation head, $f$ coefficient of friction, respectively. The fluid speed is calculated as follows:

$$
V=\frac{Q / 60}{\frac{\pi}{4} \times D^{2}}
$$

where, $\mathrm{D}$ is the inner diameter of the fluid channel. The electrical power $P$ is calculated as follows: 


$$
P=\frac{P_{d}}{\eta \cdot \cos \phi}
$$

where, $\eta$ is efficiency, $\cos \phi$ power factor. Fig. 2 is the model of the TLIM electromagnetic pump [4], Table 1 shows the material constants of liquid wood metal, and Table 2 shows the design parameters of the TLIM. In the fluid system, the TLIM produces the driving force needed to flow the liquid metal. The thrust force of the TLIM decreases with the fluid speed as shown in Fig. 3, because the eddy current decreases when the fluid speed increases. Fig. 3 shows the thrust force versus fluid speed of the TLIM and the X-mark indicates the target point of the TLIM: thrust force $40[\mathrm{~N}]$, fluid speed $0.29[\mathrm{~m} / \mathrm{s}]$. For our design value of flow rate $Q$ which is $15[1 / \mathrm{m}]$, we can calculate the pressure drop $\triangle \mathrm{P}$ by using Eq. (1) and then $\mathrm{P}_{\mathrm{d}}$ can be obtained. From the relation of $\mathrm{Pd}=\mathrm{Fx} \cdot \mathrm{V}$, Fig. 3 was obtained.

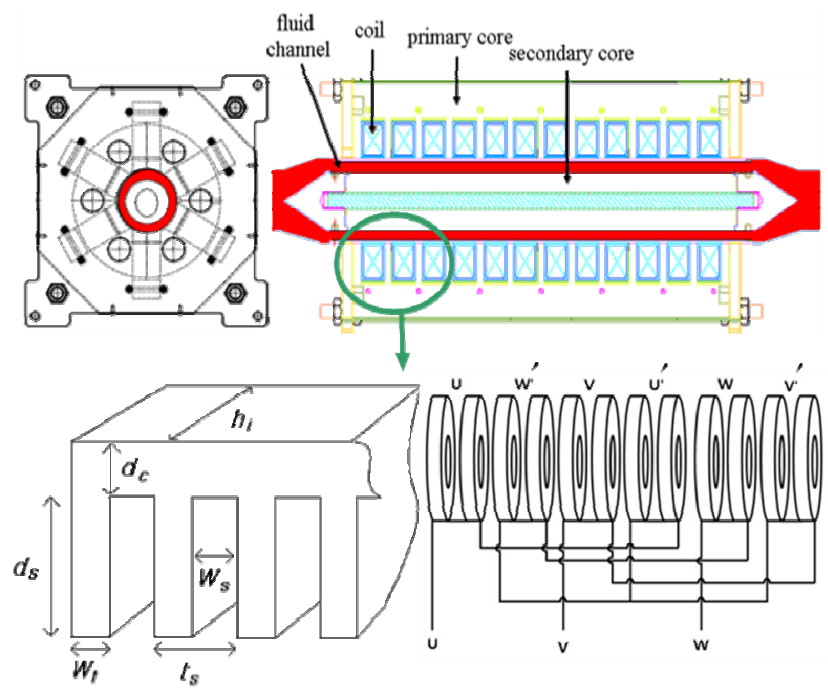

Fig. 2. Model of TLIM electromagnetic pump.

Table 1. Material constants of liquid wood metal [6]

\begin{tabular}{c|c|c}
\hline Properties & Symbol & Value $($ Unit) \\
\hline Density & $\rho$ & $9700\left(\mathrm{~kg} / \mathrm{m}^{3}\right)$ \\
\hline Viscosity & $v$ & $0.1261(\mathrm{~kg} / \mathrm{m} \cdot \mathrm{s})$ \\
\hline Melting point & $\mathrm{T}$ & $70\left({ }^{\circ} \mathrm{C}\right)$ \\
\hline Conductivity & $\sigma$ & $1.0 \times 10^{6}(\mathrm{~S} / \mathrm{m})$ \\
\hline Thermal Conductivity & $\kappa$ & $1624\left(\mathrm{~W} / \mathrm{m}^{2}\right)$ \\
\hline
\end{tabular}

Table 2. Design parameters of TLIM

\begin{tabular}{c|c|c|c}
\hline Parameter & Unit & Symbol & Value \\
\hline Slot depth & $\mathrm{mm}$ & $\mathrm{Ds}$ & 27 \\
\hline Yoke height & $\mathrm{mm}$ & $\mathrm{Dc}$ & 5 \\
\hline Stack height & $\mathrm{mm}$ & $\mathrm{Hi}$ & 31 \\
\hline Slot width & $\mathrm{mm}$ & $\mathrm{Ws}$ & 22 \\
\hline Teeth width & $\mathrm{mm}$ & $\mathrm{Wt}$ & 7 \\
\hline Slot pitch & $\mathrm{Mm}$ & $\mathrm{Ts}$ & 37.5 \\
\hline Channel diameter (inner/outer) & $\mathrm{Mm}$ & - & $46.8 / 57.2$ \\
\hline Core length & $\mathrm{Mm}$ & $\mathrm{L}$ & 348 \\
\hline
\end{tabular}

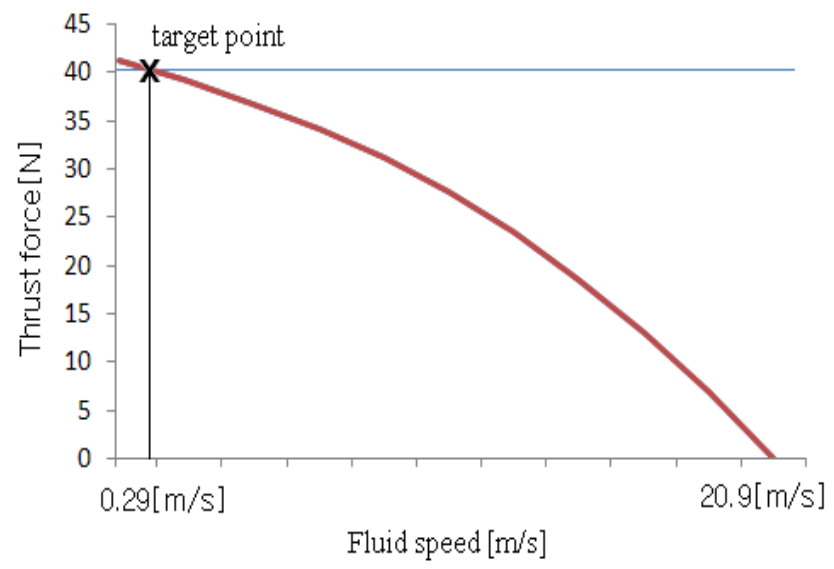

Fig. 3. Thrust force vs. fluid speed.

\section{The Analysis of Flow Characteristics}

\subsection{Fluid Flow Equations with Electromagnetic Force}

The fluid flow is driven by a TLIM located on the lower part of the system. Wood metal was used as working fluid in the experimental apparatus. The fluid flow equations considering the Lorentz force are as follows [7]:

Continuity Equation:

$$
\frac{\partial \rho}{\partial t}+\nabla \cdot(\rho \vec{V})=0
$$

Lorentz force equation:

$$
\vec{F}_{L}=\vec{J} \times \vec{B}
$$

Modified Navier-Stokes equation:

$$
\frac{\partial \vec{V}}{\partial t}+(\vec{V} \cdot \nabla) \vec{V}=-\frac{1}{\rho} \nabla P+v \nabla^{2} \vec{V}+\frac{1}{\rho} \vec{F}_{L}
$$

Where, $v$ is the kinematics viscosity of the fluid, $\vec{J}$ the current density vector, and $\vec{B}$ the magnetic field vector.

\subsection{The Algorithm for the Flow Analysis}

The velocity vector and the scalar pressure can be obtained by solving the coupled Eqs. (4) (6). In this paper, the finite volume method was used to solve the equations. The solution procedure for the equations is based on a velocity-pressure correction algorithm 'SIMPLE', SemiImplicit Method for Pressure-Linked Equation [8]. In general, a staggered grid is used to avoid the oscillation of solutions. The staggered control volume for the $\mathrm{x}$ momentum and y-momentum are shown in Figs. 4 and 5. 


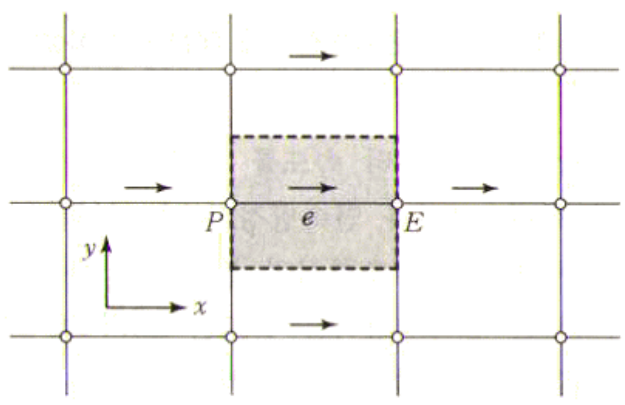

Fig. 4. Control volume for x-direction velocity.

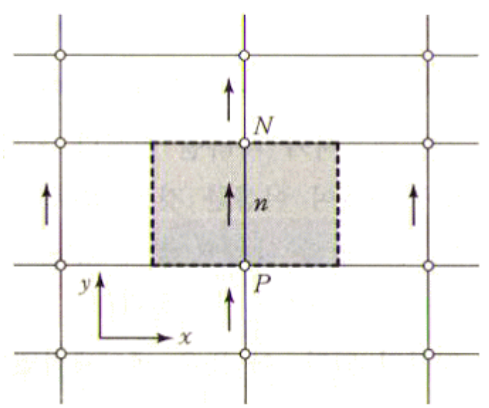

Fig. 5. Control volume for y-direction velocity.

To use the SIMPLE algorithm, discretization equations are driven from Eq. (6) as follows:

for the flow-direction,

$$
a_{e} u_{e}=\sum a_{n b} u_{n b}+b_{u}+A_{e}\left(p_{P}-p_{E}\right)
$$

and for the normal direction,

$$
a_{n} v_{n}=\sum a_{n b} v_{n b}+b_{v}+A_{n}\left(p_{P}-p_{N}\right)
$$

where, $u_{e}$ is $\mathrm{x}$-directional velocity, $v_{n}$ y-directional velocity, $b_{u}$ x-directional source term, $b_{v}$ y-directional source term including Lorentz force, $A_{e}$ x-directional control volume, $A_{n}$ y-directional control volume, $p$ pressure, $a_{n b}$ neighbor coefficients- the combined convectiondiffusion influence at the control-volume faces.

The SIMPLE algorithm suggests the pressure correction for obtaining the numerical solutions satisfied by the discretization equations. For the calculated Lorentz force and the assumed velocity and pressure, the SIMPLE iteration procedure was carried out until Eqs. (7) and (8) were satisfied.

From the flow rate of the design point, we calculated the average velocity of liquid metal fluid flowing in the annular passage of the electromagnetic pump. Then the pressure drop will be calculated from Eq. (1). By using the thrust force and fluid speed relation from Eq. (1) or using Fig. 3, the thrust force was obtained. Then the velocity and pressure distributions could be calculated by solving the modified Navier-Stokes equation using the SIMPLE algorithm.
After obtaining the velocity distribution, the corresponding flow rate could be determined until the calculated flow rate was in a small tolerance from the design point flow rate.

\subsection{MHD Flow Analysis}

Fig. 6 shows the analysis region of the annular passage flow in the TLIM. Depending on the velocity, flow can be classified into two categories: laminar (low velocity) and turbulent (high velocity) flow. For the turbulent flow, the standard k- $\varepsilon$ model was used. The flow with Lorentz force was analyzed for various flow velocities. Also, the effect of Lorentz force on the flow characteristics was investigated.

If the velocity of tube flow is low, then the flow is called laminar flow; if the flow velocity is high enough that the Reynolds number is larger than about 2,300, the flow is called turbulent flow. Fig. 7 describes the flow velocity distribution for laminar flow conditions. The mass flow rate was set to $1.0,1.5$, and $2.0 \mathrm{~kg} / \mathrm{s}$. The corresponding Reynolds numbers were much less than 2,300, which meant that the flows were laminar. Fig. 7(a) denotes the velocity distribution of the exit of annular flow passage. As the mass flow rate increased, the velocity increased with the no-slip condition at the annular tube wall. The parabolic shape - caused by the viscosity - of the laminar flow was obtained, which matched the conventional laminar flow distribution in the tube flow. Fig. 7(b) shows the vector plot of the velocity distribution.

For the turbulent flow to occur, the flow Reynolds number should be larger than 2,300. For example, a mass flow rate of $80 \mathrm{~kg} / \mathrm{s}$ corresponds to Reynolds number of 4,000 . The mass flow rates, therefore, were set to 85,90 , and $95 \mathrm{~kg} / \mathrm{s}$ for the turbulent flow conditions. Fig. 8(a) denotes the velocity distribution of the exit of the annular flow passage for the turbulent flow condition. Compared with the laminar case, this turbulent velocity is more uniformly distributed except near the wall region. Fig. 8(b) shows the vector plot of the velocity distribution for the turbulent flow. The uniformly distributed incoming velocity changed to turbulent velocity profiles.

In order to investigate the effect of magneto-hydrodynamic force on the flow characteristics of liquid metal flow, the SIMPLE algorithm including the iteration procedure was used. The final values for velocity and force were obtained: Lorentz force $40[\mathrm{~N}]$, fluid velocity $0.411[\mathrm{~m} / \mathrm{s}]$. These simulation results agree with the design values of the electromagnetic pump well. Table 3 shows the simulation

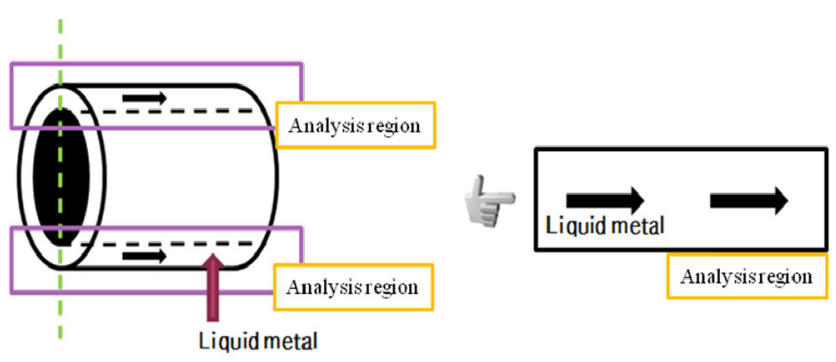

Fig. 6. Analysis region of annular passage flow. 


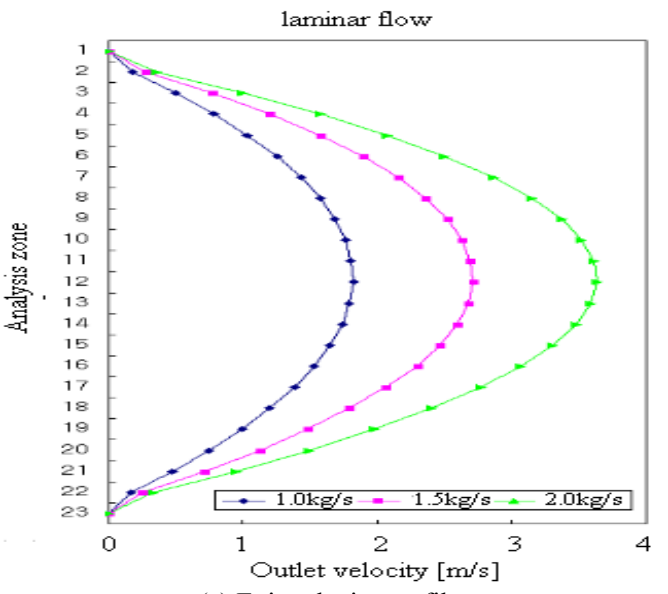

(a) Exit velocity profiles

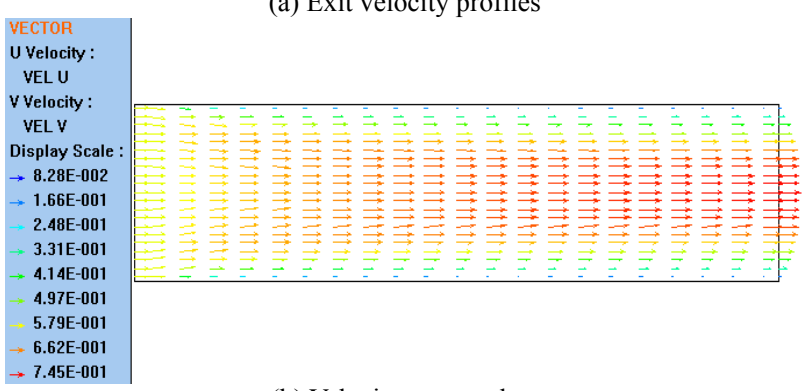

(b) Velocity vector plots

Fig. 7. Flow velocity profiles and velocity vector for laminar flow.

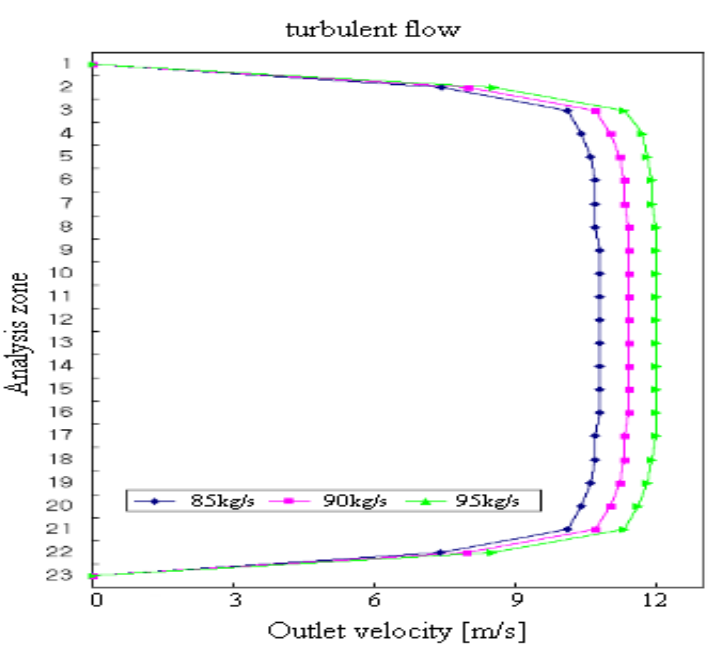

(a) Exit velocity profiles
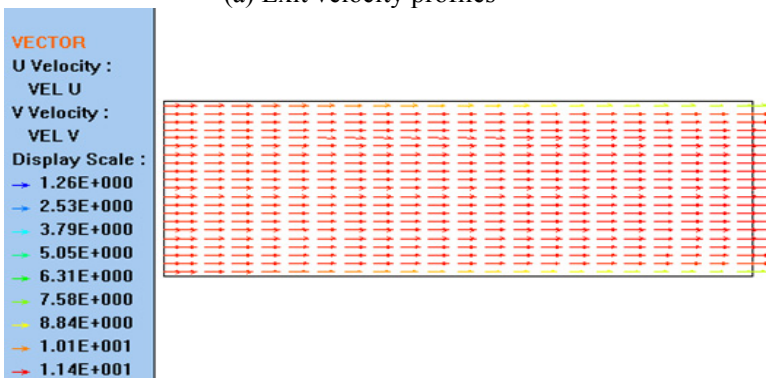

(b) Velocity vector plots

Fig. 8. Flow velocity profiles and velocity vector for turbulent flow. results for mass flow rate, velocity, and Reynolds number. Fig. 9 shows the velocity distributions of wood metal flow under various Lorentz forces: 40, 45, 55, 65 and 77[N].

Table 3. Simulation results of mass flow rate, velocity and Reynolds number

\begin{tabular}{c|c|c|c}
\hline $\mathrm{F}_{\mathrm{L}}[\mathrm{N}]$ & $\dot{m}[\mathrm{~kg} / \mathrm{s}]$ & $\mathrm{V}[\mathrm{m} / \mathrm{s}]$ & $\mathrm{Re}$ \\
\hline 40 & 0.339 & 0.411 & 164.4 \\
\hline 45 & 0.376 & 0.456 & 182.4 \\
\hline 55 & 0.448 & 0.544 & 217.6 \\
\hline 65 & 0.517 & 0.628 & 251.2 \\
\hline 77 & 0.583 & 0.708 & 283.2 \\
\hline
\end{tabular}

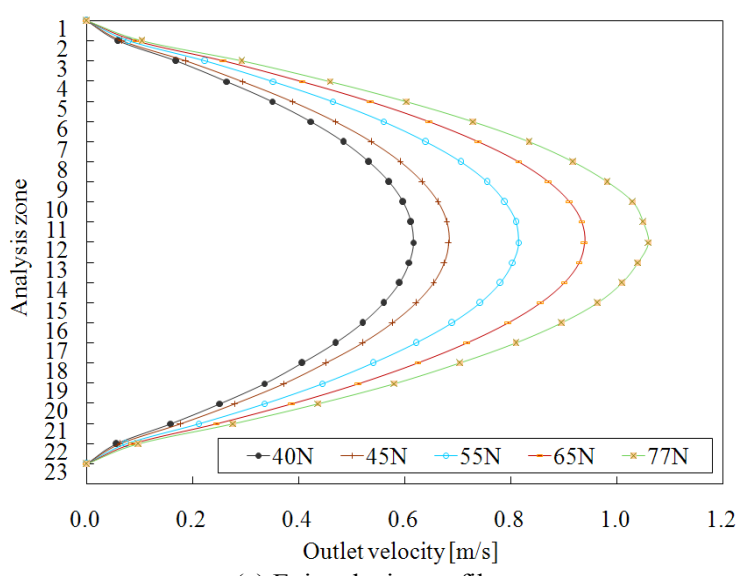

(a) Exit velocity profiles

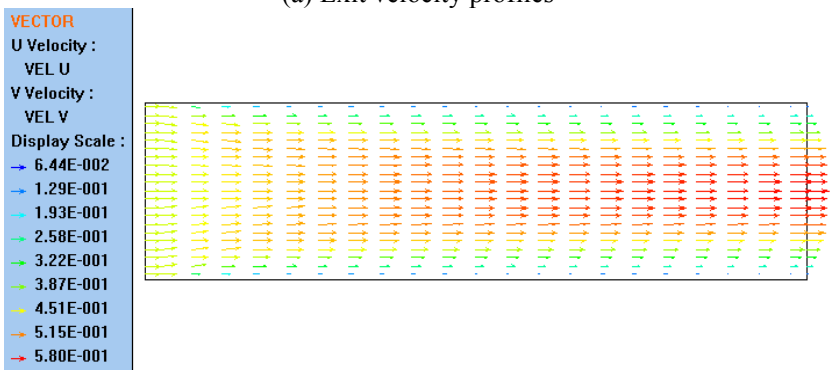

(b) Velocity vector plots

\begin{tabular}{l} 
FILL \\
VEL $U$ \\
$\begin{array}{l}0.00 E+000 \\
7.25 E-002 \\
1.45 E-001 \\
2.17 E-001 \\
2.90 E-001 \\
3.62 E-001 \\
4.35 E-001 \\
5.07 E-001 \\
5.80 E-001\end{array}$ \\
\hline
\end{tabular}

(c) Velocity distribution

Fig. 9. Flow velocity profiles, velocity vector and velocity distribution for Lorentz force $40[\mathrm{~N}]$.

\subsection{MHD Flow Rate Comparison between Simula- tion and Experiment}

Table 4 shows the comparison between the simulation and experiment for Lorentz force $40[\mathrm{~N}]$. For the experiment, we obtained the mass flow rate data by weighing the wood 
Table 4. Comparison between simulation and experiment for Lorentz force $40[\mathrm{~N}]$

\begin{tabular}{c|c|c}
\hline & Simulation & Experiment \\
\hline Average speed $[\mathrm{m} / \mathrm{s}]$ & 0.41 & 0.34 \\
\hline
\end{tabular}

metal fluid using electric balance. The simulation result showed about $17 \%$ overestimation compared with the experimental one. There are many factors which might influence the discrepancy between the simulation and experimental results. The main factors which are estimated to affect the most to the error will be the properties of the liquid wood metal such as density, viscosity, conductivity and thermal conductivity. We could not take into account the property variation by temperature due to the limited data from open literature.

\section{Conclusion}

A TLIM has been designed for a liquid metal flow system with a motor with a thrust force of 40 77 [N]. The flow characteristics have been investigated by solving the Navier-Stokes equation, where the Lorentz force was included simply by considering it as a constant in the NavierStokes equation. A wood metal was chosen to simulate the liquid metal. The effect of Lorentz forces of 40, 45, 55, 65, and $77[\mathrm{~N}]$ on the flow rate was investigated. An experiment was conducted and its results were compared with those of the simulation. The simulation result showed an overestimation of about $17 \%$ compared with the experimental one.

\section{Acknowledgements}

This work was supported by KESRI (R-2005-7-088), which is funded by MOCIE (Ministry of commerce industry and energy).

\section{References}

[1] Jacek. F. Gieras, Linear Induction Drives, Clarendon Press, 1994.

[2] S. A. Nasar, Linear motion electromagnetic systems, John Wiley and Sons, pp. 131-263, 1985.

[3] S. A. Nasar, Linear motion electric machines, John Willey and Sons, pp. 215-219, 1976.

[4] J. E. Cha, "Preliminary Design of Dynamic Corrosion-Facility for Lead-Bismuth Eutectic," KNS spring Conference, 2003.

[5] Frank M. White, Fluid Mechanics, McGraw-Hill, pp. 371-399, 2004.
[6] Keisuke Fukisaki, "Fundamental Characteristics of Molten Metal Flow Control by Linear Induction Motor," IEEE Trans. on Magnetics, Vol. 30, No. 6, pp. 4764-4766, 1994.

[7] Chuichi Arakawa, Computational Fluid Dynamics for Engineering, University of Tokyo Press, 1994.

[8] Suhas V. Patankar, Numerical heat transfer and fluid flow, McGraw-Hill, 1980.

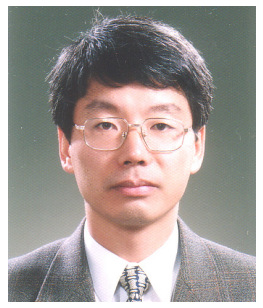

Chang-Eob Kim received his B.S. and M.S. degrees in Electrical Engineering from Seoul National University, Seoul, Korea, in 1983 and 1990, and his Ph.D. degree in Electrical Engineering from Hanyang University, Seoul, Korea, in 1995. From 1983 to 1997, he worked at Hyosung Industries Co. Ltd. as a senior researcher in the development of various motors, generators and circuit breakers. He worked as a postdoctoral fellow at the University of Southampton in the U.K. from 2000 to 2001. In 1997, he joined the Department of Electrical Engineering at Hoseo University. His teaching and research interests are the analysis of electromagnetic fields and the design of electrical machinery.

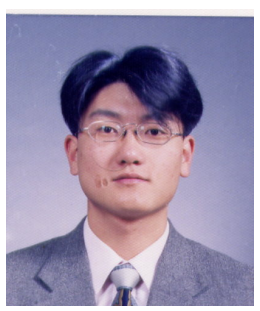

Mun Ho Jeon received his B.S., M.S. and Ph.D. degrees in Electrical Engineering from Hoseo University, Chungnam, Korea, in 2002, 2004 and 2009 , respectively. $\mathrm{He}$ is currently working as a lecturer at Hoseo University. His interests are analysis and optimum design of electrical machinery.

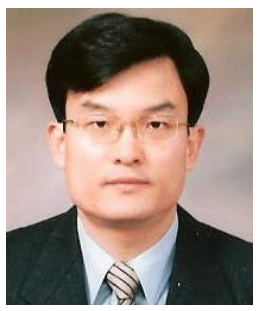

Jeong Tae Kwon received his B.S. degree in Mechanical engineering from Seoul National University, Seoul, Korea, in 1988, and his M.S. and Ph.D. degrees in Mechanical Engineering from POSTECH, Pohang, Korea, in 1993 and 1999, respectively. From 1999 to 2005, he worked at Nambu University as an assistant professor. He also worked as a postdoctoral fellow at Kyushu University in Japan from 2003 to 2004. In 2005, he joined the Department of Mechanical Engineering, Hoseo University. His teaching and research interests are the analysis of fluid flow and heat transfer in mechanical and electrical machinery. 


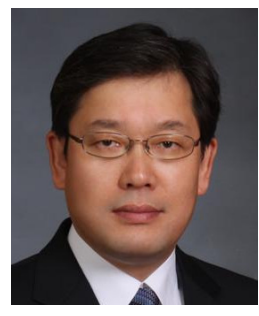

Hyo Jae Lim received his B.S. degree in Mechanical Engineering from Seoul National University, Seoul, Korea, in 1985, and his M.S. and Ph.D. degrees in Mechanical Engineering from KAIST, Seoul, Korea, in 1989 and 1995, respectively. From 1991 to 1997, he worked at Daewoo Electronic Co. Ltd. as a senior researcher in the development of household refrigerators. In 1997, he joined the department of mechanical engineering, Hoseo University. His teaching and research interests are geothermal energy and HVAC (heating, ventilation and air conditioning).

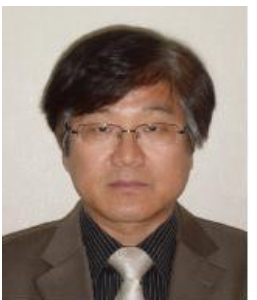

Suk Won Lee received his B.S., M.S. and Ph.D. degrees in Electrical Engineering from Seoul National University, Seoul, Korea, in 1979, 1981 and 1988, respectively. In 1990, he joined the Department of System Control Engineering, Hoseo University. His teaching and research interests are system identification and control system design using microprocessors. 